\title{
Elaboração de questionário de frequência alimentar semiquantitativo para adolescentes da região metropolitana do Rio de Janeiro, Brasil
}

\section{Development of a semiquantitative food frequency questionnaire for adolescents from the Rio de Janeiro metropolitan area, Brazil}

\section{R E S U M O}

\section{Objetivo}

Descrever a elaboração de um questionário de frequência alimentar para adolescentes do Rio de Janeiro, Brasil.

\section{Métodos}

Para seleção dos itens alimentares incluídos no questionário de frequência alimentar, utilizaram-se dados de três dias de registro alimentar obtidos em uma amostra de 430 estudantes, na faixa etária de 12 a 19 anos, da rede de ensino estadual de Niterói, Rio de Janeiro. Foram incluídos os itens alimentares mais citados nos registros e que contribuíram com 95\% do consumo de energia e nutrientes dos registros alimentares. As porções de referência foram as mais frequentemente referidas nos registros do estudo de base. As opções de frequência foram definidas de forma diferenciada para os diversos itens do questionário.

\section{Resultados}

Nos registros foram listados 306 alimentos, dos quais 249 itens foram incluídos no questionário, isoladamente ou agrupados, compondo uma lista de 90 itens. Os 14 itens alimentares mais consumidos por adolescentes do Rio de Janeiro, nesse estudo e em estudo anterior, foram associados a oito opções de frequência de

\footnotetext{
1 Universidade Federal do Rio de Janeiro, Instituto de Nutrição Josué de Castro, Centro de Ciências da Saúde, Programa de Pós-Graduação em Nutrição. Av. Carlos Chagas Filho, 373, Bloco J, $2^{\circ}$ andar, Cidade Universitária, 21941-902, Rio de Janeiro, RJ, Brasil. Correspondência para/Correspondence to: R.A. PEREIRA. E-mails: <roapereira@ufrj.br>; $<$ roapereira@gmail.com>.

2 Universidade do Estado do Rio de Janeiro, Instituto de Medicina Social, Departamento de Epidemiologia. Rio de Janeiro, RJ, Brasil.
} 
consumo; 18 itens alimentares foram acoplados a sete opções de frequência e para 58 itens foram definidas cinco opções de frequência.

\section{Conclusão}

O estudo permitiu a elaboração do questionário de frequência alimentar para adolescentes da Região Metropolitana do Rio de Janeiro, o qual será submetido às análises de reprodutibilidade e validade. Este questionário representa um instrumento valioso para pesquisas epidemiológicas que investiguem o consumo alimentar de adolescentes.

Termos de indexação: Adolescente. Consumo de alimentos. Inquéritos sobre dietas. Questionários. Registro de dieta.

\section{A B S T R A C T}

\section{Objective}

The objective of this study was to describe the development of a food frequency questionnaire for adolescents from the Rio de Janeiro metropolitan area, Brazil.

\section{Methods}

The selection of food items included in the questionnaire was done by recording the foods consumed during 3 days by 430 students aged 12 to 19 years from the public schools of Niterói (RJ) Brasil. The most cited food items, which contributed to $95 \%$ of the total energy and nutrient intakes were included in the food frequency questionnaire list. For each item, the most frequent portion size referred to in the records was defined as the reference portion. The food frequency questionnaire presented different frequency options according to the kind of food.

\section{Results}

In the dietary records, 306 food items were cited. From those, 249 were included in the food frequency questionnaire, grouped or by themselves, forming a list with 90 items. The fourteen most cited items by the adolescents from Rio de Janeiro in this and in a previous study were associated with eight frequency options; 18 and 58 other food items were associated with seven and five frequency options respectively.

\section{Conclusion}

The study enabled the development of a food frequency questionnaire for adolescents from the Rio de Janeiro metropolitan area, which will eventually be submitted to reliability and validity analysis. This food frequency questionnaire represents a valuable tool for epidemiologic investigations of food intake among adolescents.

Indexing terms: Adolescent. Food intake. Diet surveys. Questionnaire. Diet records.

\section{N T R O D U Ç Ã O}

A avaliação do consumo alimentar na fase da adolescência vem recebendo atenção, considerando, particularmente, proposições que associam hábitos alimentares inadequados em idades precoces ao desenvolvimento, na maturidade, de enfermidades como as doenças cardiovasculares, obesidade, câncer e osteoporose'.

Especialistas da Organização das Nações Unidas para Agricultura e Alimentação (FAO) e da Organização Mundial da Saúde (OMS) ressaltam as dificuldades encontradas para estimar a ingestão alimentar de crianças e adolescentes, assinalando que o método empregado para avaliar o consumo alimentar de crianças e adolescentes não deve propiciar a alteração dos hábitos alimentares usuais, seja capaz de avaliar o consumo usual e possa ser aplicado em grupos populacionais².

O Questionário de Frequência Alimentar (QFA) permite estimar o consumo de alimentos usual ao longo de um período e categorizar os indivíduos investigados segundo gradientes de consumo. Por se referir ao consumo pregresso, esse método não leva à alteração no padrão de consumo dos indivíduos e, dada a sua praticidade 
e facilidade de aplicação, o QFA vem sendo amplamente utilizado em estudos epidemiológicos desenvolvidos em longa escala ${ }^{3}$.

O QFA é um instrumento que apresenta uma lista de alimentos ou preparações e categorias de frequência de consumo estabelecidas a priori. Quando a essa lista são acopladas porções padronizadas, o questionário é chamado de semiquantitativo; nesse caso, a pergunta deve incluir a porção de referência e o consumo diário médio é estimado como um múltiplo dessa porção ${ }^{4,5}$.

O desenvolvimento do QFA destinado a adolescentes tem sido alvo de outros estudos no Brasil, onde foram desenvolvidos dois questionários de frequência alimentar para adolescentes $5^{6,7}$. Slater et al. ${ }^{6}$ validaram QFA semiquantitativo com 76 itens para adolescentes de São Paulo; esse questionário foi elaborado a partir de estudo realizado com 200 adolescentes estudantes de uma instituição particular de ensino. Carvalho et al. ${ }^{7}$ desenvolveram QFA com 34 itens alimentares para avaliar o consumo alimentar de adolescentes de Teresina, Piauí.

Dada a ausência de QFA específico para adolescentes do Rio de Janeiro, o consumo alimentar nesse grupo tem sido analisado a partir da aplicação de QFA validado para população adulta do Rio de Janeiro ${ }^{8-10}$. Considera-se, entretanto, que o consumo dos adolescentes se diferencia do consumo familiar e que o desenvolvimento de instrumentos elaborados especificamente para esse grupo etário deve ser tratado como uma prioridade para o acompanhamento da situação alimentar dos adolescentes do Rio de Janeiro ${ }^{11 .}$.

Este artigo descreve o processo aplicado na elaboração de QFA destinado a adolescentes da Região Metropolitana do Rio de Janeiro.

\section{MÉ TO D O S}

Para selecionar os alimentos que compõem o QFA, utilizaram-se os dados de registro de alimentos obtidos em estudo transversal de base escolar sobre fatores de risco para doenças cardiovasculares, realizado entre junho e dezembro de 2003, com adolescentes de 12 a 18,9 anos, estudantes da rede de ensino estadual da cidade de Niterói (RJ). Nesse estudo, foi selecionada amostra probabilística por conglomerados com dois estágios de seleção (escolas e turmas) estimada em 600 estudantes, dos quais 430 (72\% da amostra necessária para o estudo) completaram três registros alimentares: dois dias durante a semana e um dia de final de semana.

Para definir os alimentos incluídos no QFA, todos os itens alimentares citados nos registros foram organizados em ordem decrescente de frequência. Em seguida, tratou-se de agrupar os itens similares, como por exemplo: biscoitos de amido de milho, de milho, de fécula de batata, de leite e de coco foram agrupados no item alimentar "biscoito doce simples". A categoria dos biscoitos foi completada com os biscoitos salgados e biscoitos recheados. Outro exemplo foi o item alimentar incluído no questionário, "carne de boi", que agrupou os seguintes alimentos citados nos registros: bife, carne assada, moída, ensopada, almôndega e espetinho de carne. Para compor a lista do questionário foram selecionados os itens referidos no mínimo 15 vezes nos registros.

Aplicou-se, paralelamente, a metodologia proposta por Block et al. ${ }^{12}$. Para tanto, estimou-se o consumo total relatado em todos os registros alimentares de energia, proteína, glicídios, lipídeos, colesterol, vitamina A, vitamina $C$, ferro e cálcio. Em seguida, foi calculada a contribuição percentual de cada um dos alimentos relatados nos registros para o consumo total de energia e nutrientes. Finalmente, esses alimentos foram listados em ordem decrescente de contribuição percentual para o consumo de energia e nutrientes, estimando também a contribuição acumulada. Os alimentos que contribuíram para 95\% do consumo total de energia, macronutrientes, colesterol, vitaminas A e C, ferro e cálcio foram selecionados para compor a lista do QFA.

Para determinar porções de referência dos itens alimentares incluídos no QFA, utilizaram-se 
as "porções naturais" ou "típicas" de alimentos como: pão francês, banana, laranja, maçã, ovo, cachorro-quente, hambúrguer. Para alimentos cujo porcionamento não se dá de forma tão natural, como é o caso do arroz, feijão e carnes, foram consideradas as porções mais frequentemente referidas nos registros do estudo de base ou as medianas das distribuições das quantidades relatadas nos registros transformadas em medidas caseiras. As informações sobre medidas caseiras foram complementadas com aquelas mais citadas para servir alimentos em enquete sobre utensílios domésticos ${ }^{13}$.

Quanto às opções de frequência foram adotadas as utilizadas em QFA validado para adultos no Rio de Janeiro ${ }^{10}$ e considerando a proposta de questionário desenvolvido para adolescentes nos Estados Unidos por Rockett et al. ${ }^{14}$. Após pré-teste, o questionário foi definido no formato vertical e as opções de frequência foram organizadas de forma diferenciada para determinados itens do questionário.

O QFA é um instrumento elaborado para estimar o consumo alimentar usual médio ao longo de um período, que é definido como o período de referência. Neste QFA, o período de referência foram os seis meses precedentes à entrevista. Assim, o adolescente foi perguntado com que frequência cada alimento foi consumido, tipicamente, nos últimos seis meses. $O$ anexo exemplifica a estrutura do QFA elaborado.

Em uma seção preliminar do QFA, foram integradas perguntas abordando informações sobre hábitos de alimentação, como o uso de açúcar, adoçante, azeite de oliva, tipo de leite usualmente consumido, consumo de frituras, pele de frango, gordura aparente das carnes, local de refeições, hábito de "beliscar" entre as refeições e uso de suplementos vitamínicos.

A pesquisa que gerou os dados para a elaboração do questionário foi aprovada pelo Comitê de Ética em Pesquisa do Hospital Universitário Clementino Fraga Filho, da Universidade
Federal do Rio de Janeiro, em 11/6/02 (Protocolo $n^{\circ}$ 041/02). A participação na pesquisa foi condicionada à assinatura do Termo de Consentimento Livre e Esclarecido por responsável ou pelo indivíduo com idade igual ou maior de 18 anos.

\section{RESULTADOS}

Nos registros dos adolescentes de Niterói foram listados 306 alimentos, dos quais 249 itens foram agrupados em uma lista de 90 itens alimentares que compuseram o QFA. O alimento referido com maior frequência foi o arroz, com 1207 citações, seguido do feijão, pão francês, leite, carne de boi, refrigerante, achocolatado, café e açúcar. Dentre os itens mais frequentemente referidos pelos adolescentes, observou-se a presença marcante de produtos industrializados e com alta densidade energética e baixo valor nutricional, destacando-se: refrigerante, achocolatado, açúcar, sucos industrializados, balas, biscoitos recheado e salgado, manteiga, margarina e salgados (Quadro 1).

Ao se cotejar a lista de itens alimentares elaborada por ordem de citação e a desenvolvida por contribuição percentual ao consumo de energia e nutrientes, observou-se que a primeira contemplava todos os itens que apresentaram contribuição substancial (95\% do consumo estimado) para o consumo dos nutrientes investigados.

Sessenta e três alimentos foram mencionados menos de 15 vezes nos registros. Entre esses, foram mantidos no QFA: peixe enlatado, bebidas alcoólicas e refrigerantes de baixa caloria.

Para 14 itens alimentares - leite, café, pão francês, margarina, arroz, feijão, entre outros que figuraram entre os alimentos mais consumidos entre adolescentes do Rio de Janeiro em estudo realizado em 1995-1996', foram determinadas oito opções de frequência, que variaram de "menos de uma vez por mês ou nunca" a "quatro ou mais vezes ao dia". Outros 18 itens foram associados a sete opções de frequência que variaram de "menos de uma vez por mês ou 
Quadro 1. Itens alimentares selecionados para compor a lista de questionário de frequência alimentar elaborado para adolescentes do Rio de Janeiro e o número de citações nos registros alimentares de adolescentes de Niterói (RJ), 2003.

\begin{tabular}{|c|c|c|c|c|c|}
\hline Itens alimentares & Citações (n) & Itens alimentares & Citações (n) & Itens alimentares & Citações (n) \\
\hline Arroz & 1207 & Biscoito doce simples & 168 & Chá ou mate & 46 \\
\hline Feijão & 1020 & Pão doce & 152 & Bolo com cobertura & 45 \\
\hline Pão francês & 773 & Pipoca & 151 & Molhos & 44 \\
\hline Leite & 758 & Molho de maionese & 144 & Vísceras & 43 \\
\hline Carne de boi & 666 & Tomate & 142 & Sopa industrializada & 42 \\
\hline Refrigerante & 582 & Filé de frango & 141 & Aipim/inhame & 41 \\
\hline Achocolatado & 520 & Suco natural & 139 & Beterraba & \multirow{2}{*}{39} \\
\hline Café & 506 & Chocolate/bombom & 106 & Chuchu & \\
\hline Açúcar & 496 & Doce de fruta & 105 & Carne seca & 38 \\
\hline Batata & 493 & Sanduíche & 95 & Bacon & 35 \\
\hline Suco industrializado & 456 & Cenoura & \multirow{2}{*}{92} & Couve-flor ou brócolis & 35 \\
\hline Bala & 428 & Peixe & & Creme de leite & 34 \\
\hline Biscoito recheado & 393 & Maçã & 86 & Abóbora & \multirow{2}{*}{32} \\
\hline Queijo & 360 & Amendoim & 85 & Morango & \\
\hline Margarina & 311 & logurte & \multirow{2}{*}{75} & Polenta & 29 \\
\hline Biscoito salgado & 302 & Panqueca & & Lasanha & \multirow{2}{*}{26} \\
\hline Manteiga & & Laranja & 71 & Mamão & \\
\hline Presunto/mortadela & 292 & Mingau & 70 & Gelatina & 22 \\
\hline Salgado & 285 & Pizza & 69 & Nuggets & \multirow{2}{*}{21} \\
\hline Macarrão & 283 & Requeijão & 68 & Picolé & \\
\hline Bolo simples & 269 & Hambúrguer & 63 & Azeite de oliva & \multirow{3}{*}{19} \\
\hline Linguiça/salsicha & 252 & Repolho/couve & 62 & Milho verde & \\
\hline Ovo & 213 & Chips (salgadinhos) & 60 & Molho de catchup & \\
\hline Frango & 210 & Porco & 59 & Quiabo & \multirow[t]{2}{*}{18} \\
\hline Alface/agrião & 204 & Sorvete & 53 & Complementos nutricionais & \\
\hline Banana & 194 & Cebola & \multirow{2}{*}{50} & Vinho & \multirow[t]{2}{*}{16} \\
\hline Pão de forma & 191 & Produtos à base de cereais & & Abacaxi & \\
\hline Doce de leite & 182 & Cachorro-quente & 49 & Cerveja & \multirow{3}{*}{15} \\
\hline Salgadinhos & 174 & Macarrão instantâneo & 48 & Goiaba & \\
\hline & & & & Pepino & \\
\hline
\end{tabular}

nunca" a "duas ou mais vezes por dia", por exemplo: achocolatado em pó, iogurte, pão doce, queijo, laranja, ovo, etc., e para 58 itens as cinco opções de frequências definidas variaram de "menos de uma vez por mês ou nunca" a "cinco ou mais vezes por semana" (Quadro 2).

Para aqueles alimentos que não apresentam porção "natural" ou "típica", foram adotadas como porção de referência as porções medianas das distribuições das quantidades relatadas nos registros do estudo de base, as quais foram transformadas em medidas caseiras, como descrito no Quadro 3. Exemplificando: para o arroz a porção de referência adotada no QFA foi "uma colher de servir", que corresponde à mediana das quantidades de arroz relatadas nos registros.
D I S C U S S Ã O

Elaborou-se QFA com 90 itens alimentares destinado a estimar o consumo de alimentos de adolescentes da Região Metropolitana do Rio de Janeiro, no qual a lista de alimentos e as respectivas porções de referência foram compiladas a partir de informações de registros alimentares de adolescentes da mesma área. O QFA proposto inclui os alimentos mais citados nos registros alimentares obtidos no estudo de base e aqueles que forneceram $95 \%$ do consumo de energia e nutrientes selecionados relatado nesses registros, incluindo também alimentos que possivelmente são discriminantes ou indicam tendências do consumo alimentar desse grupo. 
Quadro 2. Opções de frequência associadas aos itens alimentares de QFA elaborado para adolescentes do Rio de Janeiro (RJ), 2003.

\begin{tabular}{|c|c|}
\hline Itens alimentares & Opção de frequência do questionário \\
\hline $\begin{array}{l}\text { Leite; chá ou mate; café; pão de forma; pão francês; margarina; manteiga; feijão; } \\
\text { arroz; refrigerante light ou dietético; refrigerante; suco de fruta natural; suco indus- } \\
\text { trializado e bala }\end{array}$ & $\begin{array}{l}\text { Menos de uma vez por mês ou nunca } \\
1-3 \text { vezes por mês } \\
1 \text { vez por semana } \\
2-4 \text { vezes por semana } \\
5-6 \text { vezes por semana } \\
1 \text { vez por dia } \\
2-3 \text { vezes por dia } \\
4 \text { ou mais vezes por dia }\end{array}$ \\
\hline $\begin{array}{l}\text { Achocolatado em pó; iogurte; produtos à base de cereais; sustagem e outros } \\
\text { complementos em pó; mingau ou canjica; pão doce; biscoito cream cracker ou outro } \\
\text { biscoito salgado; biscoito doce simples; biscoito recheado ou wafer; requeijão; } \\
\text { queijo; ovo ou omelete; laranja ou tangerina; banana; alface ou agrião; tomate; } \\
\text { batata e salgados }\end{array}$ & $\begin{array}{l}\text { Menos de uma vez por mês ou nunca } \\
1-3 \text { vezes por mês } \\
1 \text { vez por semana } \\
2-4 \text { vezes por semana } \\
5-6 \text { vezes por semana } \\
1 \text { vez por dia } \\
2 \text { ou mais vezes por dia }\end{array}$ \\
\hline $\begin{array}{l}\text { Pão de queijo; bolo simples; bolo com cobertura e/ou recheio; macarrão instantâ- } \\
\text { neo; macarrão cozido; lasanha; panqueca, nhoque, torta salgada, empadão e } \\
\text { outras massas; polenta, angu, cuscuz salgado; farinha de mandioca ou farofa; } \\
\text { sopas industrializadas; frango; carne de porco; peixe enlatado; peito de frango; } \\
\text { linguiça ou salsicha; carne de boi; fígado, dobradinha ou outra víscera; peixe; carne } \\
\text { seca ou outra carne salgada; bacon; presunto, mortadela, apresuntado, etc.; mo- } \\
\text { rango; abacaxi; maçã; goiaba; couve-flor ou brócolis; mamão; chuchu; repolho; } \\
\text { quiabo; pepino; beterraba; cenoura; cebola; aipim ou inhame; milho verde; abóbo- } \\
\text { ra; sorvete ou picolé; doce de leite, pudim, leite condensado ou brigadeiro; pipoca; } \\
\text { gelatina; chocolate ou bombom; cachorro-quente; doce de fruta; hambúrguer; } \\
\text { amendoim, doce de leite ou paçoca; batata frita; batata chips; pizza; nuggets; } \\
\text { sanduíche; molho de maionese ou outros molhos cremosos para salada; molho } \\
\text { catchup; outros molhos industrializados, como mostarda, molho branco, molho } \\
\text { bolonhesa, etc.; creme de leite; cerveja; vinho e pinga, cachaça, uísque, conhaque, } \\
\text { coquetéis com álcool e outras bebidas }\end{array}$ & $\begin{array}{l}\text { Menos de uma vez por mês ou nunca } \\
1-3 \text { vezes por mês } \\
1 \text { vez por semana } \\
2-4 \text { vezes por semana } \\
5 \text { ou mais vezes por semana }\end{array}$ \\
\hline
\end{tabular}

Quadro 3. Porções de referência adotadas em QFA desenvolvido para adolescentes do Rio de Janeiro (RJ), 2003.

\begin{tabular}{|c|c|}
\hline Itens alimentares & Porções de referência \\
\hline Leite; chá ou mate; café & 1 copo de requeijão ou uma xícara de café \\
\hline $\begin{array}{l}\text { Achocolatado; sustagem ou outros complementos similares em pó; polenta, angu ou } \\
\text { cuscuz salgado; farinha de mandioca ou farofa; bacon; cebola; doce de leite, pudim, } \\
\text { leite condensado, ou brigadeiro; doce de fruta }\end{array}$ & 1 colher de sopa \\
\hline logurte & 1 copo ou pote \\
\hline Produtos à base de cereais & $1 / 2$ xícara ou 3 colheres de sopa \\
\hline Mingau ou canjica; pipoca & 1 prato fundo \\
\hline Margarina; manteiga & 1 ponta de faca ou uma colher de chá \\
\hline Bolo simples ou com cobertura e/ou recheio; queijo & 1 fatia ou pedaço médio \\
\hline Requeijão & 1 colher de sobremesa \\
\hline Feijão & 1 concha \\
\hline Arroz; batata; abóbora & 1 colher de servir \\
\hline Macarrão cozido & 1 pegador \\
\hline $\begin{array}{l}\text { Carne de boi; fígado, dobradinha ou outra víscera; carne seca ou outra carne salgada; } \\
\text { alface; chuchu; repolho ou couve; quiabo; pepino; beterraba; cenoura; tomate }\end{array}$ & 3 colheres de sopa \\
\hline Refrigerante light ou dietético; refrigerante; suco de fruta natural; suco industrializado & 1 copo de requeijão \\
\hline
\end{tabular}


O QFA deve ser específico para a população que se deseja estudar, uma vez que a lista dos alimentos deve ser baseada no consumo habitual dessa população. De acordo com Costa et al. ${ }^{15}$, a definição da lista de alimentos do QFA depende do que é planejado para a aplicação do QFA. Quando o objetivo é investigar a ingestão de um nutriente específico, a lista de alimentos deve incluir itens com elevado conteúdo desse nutriente que sejam parte do hábito alimentar da população estudada. Contudo, se o objetivo é descrever o consumo alimentar geral é importante que a lista seja constituída por um número abrangente de alimentos que sejam representativos do consumo habitual da população a ser investigada ${ }^{3,4}$.

Na seleção dos itens alimentares para compor a lista do questionário pode-se partir de informações de tabelas de composição de alimentos, da orientação de especialistas em alimentos e nutrição, ou de dados de estudos anteriores que aplicaram registro ou recordatório de 24 horas na população que se deseja investigar ${ }^{3}$, selecionando aqueles mais informativos sobre o consumo alimentar do grupo em estudo, particularmente os que apresentam alta variabilidade interpessoal ${ }^{4}$.

Barbosa et al. ${ }^{11}$ salientaram que o QFA é um instrumento apropriado para avaliar o consumo alimentar usual de adolescentes e o formato vertical tem sido considerado o mais adequado para a aplicação em crianças, idosos e populações com baixa escolaridade ${ }^{3}$; nesse caso as opções de frequência são apresentadas separadas para cada alimento, o que permite a designação de opções de frequência distintas para os itens alimentares.

Cade et al. ${ }^{5}$ assinalam que a inclusão de categorias de frequência e tamanhos das porções no QFA reduz o tempo de codificação, transcrição de erros e também o número de questionários a serem rejeitados, devido a respostas incompletas ou em branco. Esses autores apontam que as opções de frequência podem ser as mesmas para todos os alimentos do QFA ou podem variar de acordo com o alimento analisado. A adoção de opções de frequência diferenciadas para os diversos itens do QFA justifica-se pelo fato de que há um número restrito de alimentos que são consumidos mais que do que duas vezes por dia, enquanto outros são consumidos diversas vezes no dia (por exemplo, açúcar e outro itens de adição, pães, biscoitos etc.). Assim, se o questionário apresentar opções de frequência de consumo "muitas vezes ao dia" uniformemente para todos os alimentos, pode haver tendência à superestimação do relato do consumo para aqueles itens que não são usualmente consumidos diversas vezes no dia ${ }^{5}$.

No Brasil, já foram desenvolvidos QFA para adultos do Rio de Janeiro ${ }^{10}$, Viçosa ${ }^{16}$ e Brasília ${ }^{17}$, adolescentes de Teresina ${ }^{7}$, trabalhadores de baixa renda de Goiânia ${ }^{18}$ e estudo de caso-controle sobre dieta e câncer de mama em mulheres de João Pessoa ${ }^{19}$. Particularmente no estado de São Paulo, foram desenvolvidos QFA para adolescentes6, crianças de dois a cinco anos de idade ${ }^{20}$ e de cinco a dez anos de idade ${ }^{21}$, mulheres de ascendência japonesa ${ }^{22}$, adultos com excesso de peso ${ }^{23}$, para estudo sobre a relação entre dieta e doenças crônicas $^{24}$ e dieta e câncer oral e de laringe ${ }^{25}$. Esses questionários apresentavam listas de alimentos que incluíam de 21 a 127 itens.

Slater et al. ${ }^{6}$ desenvolveram e validaram um QFA semiquantitativo específico para avaliar o consumo alimentar de adolescentes de São Paulo. Similarmente ao relatado neste estudo, os autores elaboraram a lista de alimentos do QFA a partir de estudo no qual foram obtidos dois registros alimentares de 200 adolescentes, de ambos os sexos, de uma instituição particular de ensino de São Paulo. Os alimentos relatados nos registros foram agrupados de acordo com suas características físicas e valores nutricionais em 140 itens alimentares. A partir dessa lista foram identificados os alimentos que mais contribuíram para o consumo de energia e nutrientes, sendo definida uma lista final com 76 itens alimentares. Tal como no QFA desenvolvido para os adolescentes do Rio de Janeiro, o período de referência para a estimativa 
do consumo usual de alimentos foram os seis meses precedentes à entrevista. Diferentemente do QFA elaborado para adolescentes do Rio de Janeiro, o QFA desenvolvido para adolescentes de São Paulo apresentava uma lista de alimentos mais reduzida, formato horizontal e não previa o emprego de opções de frequência de consumo diferenciadas para os diversos itens alimentares.

Carvalho et al. ${ }^{7}$ desenvolveram um QFA qualitativo com a finalidade de avaliar o consumo alimentar de adolescentes de Teresina, Piauí. O instrumento foi estruturado em três grupos: alimentos construtores, alimentos reguladores e alimentos energéticos. O QFA foi elaborado com 34 itens alimentares e o consumo dos alimentos foi quantificado somente pela frequência relatada pelos adolescentes.

No município do Rio de Janeiro, foi validado um QFA semiquantitativo destinado a avaliar o consumo habitual da população adulta no ano anterior ao seu preenchimento ${ }^{10}$. Esse instrumento inclui 81 itens alimentares e duas ou três porções de referência associadas aos itens alimentares. Embora venha sendo empregado em estudos com adolescentes ${ }^{8,9}$, esse QFA não foi validado para esse grupo populacional e entre os alimentos listados não estão incluídos alguns dos que foram frequentemente citados no estudo que embasou a elaboração do presente questionário, como por exemplo: suco industrializado, nuggets, doce de fruta, amendoim, doce e paçoca de amendoim, molho de catchup, pão de queijo, entre outros.

Tais observações evidenciam a necessidade de elaboração de um instrumento específico para adolescentes do Rio de Janeiro, particularmente, levando em conta que a importância das práticas alimentares nas idades precoces vem sendo enfatizada tendo em vista a prevenção de agravos à saúde ao longo da vida, principalmente no caso de doenças crônicas não transmissíveis e transtornos alimentares ${ }^{26,27}$.

Considerando que a elaboração da lista de alimentos do QFA aqui descrita foi fundamentada em estudo que analisou amostra repre- sentativa de adolescentes, o instrumento proposto é um questionário que permitirá avaliar o consumo usual global de adolescentes da Região Metropolitana do Rio de Janeiro. Esse QFA constitui um instrumento que poderá ser empregado para acompanhar a evolução do consumo de alimentos de adolescentes e a avaliação de propostas de intervenção voltadas para esse grupo na região de estudo.

Neste trabalho foram descritas as etapas iniciais do processo de construção desse QFA. O instrumento foi elaborado com base em dados obtidos em amostra representativa de adolescentes de um município da Região Metropolitana do Rio de Janeiro e na sua definição foram empregados procedimentos bem definidos e consagrados na literatura. Esse instrumento, elaborado criteriosamente com base nas recomendações de especialistas, certamente será de utilidade para a pesquisa sobre saúde e nutrição de adolescentes. O processo de elaboração do questionário ainda inclui diversas etapas igualmente importantes para a obtenção de um instrumento válido ainda serão concretizadas. O QFA será ainda avaliado do ponto de vista da linguagem e da forma, e será acoplado a um conjunto de fotografias que poderão facilitar e melhorar a estimativa da quantidade consumida e torná-lo um instrumento autopreenchível e, também, será analisado quanto a sua confiabilidade e validade.

\section{A GRADECIMENTOS}

Ao conselho Nacional de Desenvolvimento Científico e Tecnológico (processo n 506336/2004-2) e à Dra. Vera Lúcia Chiara, pela colaboração na redação inicial do manuscrito.

\section{COLABORADORES}

M.C. ARAUJO participou na elaboração do instrumento, na análise e na interpretação dos dados e na redação do artigo. G.V. VEIGA foi responsável pela investigação que forneceu os dados de registro de alimentos que permitiram a elaboração do instru- 
mento e contribuiu para a forma final do artigo. $\mathrm{R}$. SICHIERI participou na concepção do projeto e contribuiu na análise dos dados. R.A. PEREIRA foi responsável pela concepção do projeto, pela elaboração do instrumento, pela análise e pela interpretação dos dados e pela redação do artigo.

\section{REFERÊ NCIAS}

1. Anding JD, Kubena KS, McIntosh WA, O'Brien B. Blood lipids, cardiovascular fitness, obesity, and blood pressure: the presence of potential coronary heart disease risk factors in adolescents. J Am Diet Assoc. 1996; 96(3):238-42. doi:10.1016/500028223(96)00073-9.

2. World Health Organization. Energy and protein requirements. Geneve: WHO; 1985. Technical Report Series, 724.

3. Willett $W C$, editor. Nutritional epidemiology. $2^{\text {nd }}$ ed. Oxford: Oxford University Press; 1998.

4. Fisberg RM, Slater B, Marchioni DML, Martini LA, editores. Inquéritos alimentares: métodos e bases científicos. São Paulo: Manole; 2005.

5. Cade J, Thompson R, Burley V, Warm D. Development, validation and utilization of foodfrequency questionnaires: a review. Public Health Nutr. 2002; 5(4):567-87. doi:10.1079/PHN2001 318.

6. Slater B, Philippi ST, Fisberg RM, Latorre MRDO. Validation of a semi-quantitative adolescent food frequency questionnaire applied at a public school in São Paulo, Brazil. Eur J Clin Nutr. 2003; 57(5): 629-35. doi: 10.1038/sj.ejcn. 1601588.

7. Carvalho CMRG, Nogueira AMT, Teles JBM, Paz SMR, Souza RML. Consumo alimentar de adolescentes matriculados em um colégio particular de Teresina, Piauí, Brasil. Rev Nutr. 2001; 14(2):85-93. doi: 10.1590/S1415-52732001000200001.

8. Chiara VL, Sichieri R. Consumo alimentar em adolescentes. Questionário simplificado para avaliação de risco cardiovascular. Arq Bras Cardiol. 2001; 77(4):332-6. doi: 10.1590/S0066-782X2001 001000003.

9. Andrade RG, Pereira RA, Sichieri R. Consumo alimentar de adolescentes com e sem sobrepeso do município do Rio de Janeiro. Cad Saúde Pública. 2003; 19(5):1485-95. doi: 10.1590/S0102-311X 2003000500027.

10. Sichieri R, Everhart JE. Validity of a Brazilian food frequency questionnaire against dietary recalls and estimated energy intake. Nutr Res. 1998; 18(10): 1649-59. doi: 10.1016/S0271-5317(98)00151-1.
11. Barbosa KBF, Rosado LEFPL, Franceschini SCC, Priore SE. Instrumentos de inquérito dietético utilizados na avaliação do consumo alimentar em adoles centes: comparação entre métodos. Arc Latinoam Nutr. 2007; 57(1):43-50.

12. Block G, Hartman AM, Dresser CM, Carroll MD, Gannon J, Gardner L. A data-based approach to diet questionnaire design and testing. Am J Epidemiol. 1986; 124(3):453-69.

13. Araújo MC, Melo LPC, Ribeiro RL. Enquete sobre utensílios mais utilizados para servir alimentos. Livro de resumos da XXVII Jornada Giulio Massarani de Iniciação Científica, Artística e Cultural da Universidade Federal do Rio de Janeiro, 2005. Rio de Janeiro: Universidade Federal do Rio de Janeiro; 2005. p.304.

14. Rockett HRH, Breitenbach M, Frazier L, Witschi J, Wolf $A M$, Field $A E$, et al. Validation of a youth/ adolescent food frequency questionnaire. Prev Med.1997; 26(6):808-16.

15. Costa AGV, Priore SE, Sabarense CM, Franceschini SCC. Questionário de frequência de consumo alimentar e recordatório de 24 horas: aspectos metodológicos para avaliação da ingestão de lipídeos. Rev Nutr. 2006; 19(5):631-41. doi: 10.15 90/S1415-52732006000500002.

16. Crispim SP. Validação relativa de um questionário semiquantitativo de frequência alimentar para avaliar a ingestão dietética de adultos - Viçosa Minas Gerais [dissertação]. Visçosa: Universidade Federal de Viçosa; 2004.

17. Ribeiro AC, Sávio KEO, Rodrigues MLCF, Costa THM, Schmitz BAS. Validação de um questionário de frequência de consumo alimentar para população adulta. Rev Nutr. 2006;19(5):553-62. doi: 10.1590/S1415-52732006000500003.

18. Fornés NS, Stringhini MLF, Elias BM. Reproducibility and validity of a food-frequency questionnaire for use among low-income Brazilian workers. Public Health Nutr. 2003; 6(8):821-7. doi: 10.1079/PHN 2003505 .

19. Lima FEL, Fisberg RM, Slater B. Desenvolvimento de um questionário quantitativo de frequência alimentar (QQFA) para um estudo caso-controle de dieta e câncer de mama em João Pessoa - PB. Rev Bras Epidemiol. 2003; 6(4):373-79. doi: 10.15 90/S1415-790X2003000400011.

20. Colluci ACA, Philippi ST, Slater B. Desenvolvimento de um questionário de frequência alimentar de crianças de 2 a 5 anos de idade. Rev Bras Epidemiol. 2004; 7(4):393-401. doi: 10.1590/S1415-790X20 04000400003.

21. Fumagalli F, Monteiro JP, Sartorelli DS, Vieira MNCM, Bianchi MLP. Validation of a food 
frequency questionnaire for assessing dietary nutrients in Brazilian children 5 to 10 years of age. Nutrition. 2008; 24(5):427-32. doi: 10.1016/j.nut. 2008.01.008.

22. Cardoso MA, Kida AA, Tomita LY, Stocco PR. Reproducibility and validity of a food frequency questionnaire among women of Japanese ancestry living in Brazil. Nutr Res. 2001; 21(5):725-33. doi:10.1016/S0271-5317(01)00283-4.

23. Salvo VLMA, Gimeno SGA. Reprodutibilidade e validade do questionário de frequência de consumo de alimentos. Rev Saúde Pública. 2002; 36(4): 505-12. doi: 10.1590/S0034-8910200200040 0018.

24. Furlan-Viebig R, Pastor-Valero M. Desenvolvimento de um questionário de frequência alimentar para o estudo de dieta e doenças não transmissíveis. Rev Saúde Pública. 2004; 38(4):581-4. doi: 10.15 90/S0034-89102004000400016.
25. Matarazzo HCZ, Marchioni DML, Figueiredo RAO, Slater B, Neto JE, Filho VW. Reprodutibilidade e validade do questionário de frequência de consumo alimentar utilizado em estudo caso-controle de câncer oral. Rev Bras Epidemiol. 2006; 9(3): 316-24. doi:10.1590/S1415-790X2006000300 006.

26. Austin SB, Ziyadeh NJ, Forman S, Prokop LA, Keliher $A$, Jacobs D. Screening high school students for eating disorders: results of a national initiative. Prev Chronic Dis. 2008; 5(4):A114.

27. Lake AA, Mathers JC, Rugg-Gunn AJ, Adamson AJ. Longitudinal change in food habits between adolescence (11-12 years) and adulthood (32-33 years): the ASH30 Study. J Public Health. 2006; 28(1):10-16.

Recebido em: 13/3/2008

Versão final reapresentada em: 17/8/2009 Aprovado em: 11/11/2009 


\section{ANEXO}

EXEMPLO DA ESTRUTURA DE QUESTIONÁRIO DE FREQUÊNCIA ALIMENTAR DESENVOLVIDO PARA ADOLESCENTES DO RIO DE JANEIRO (RJ).

Com que frequência você comeu estes alimentos nos últimos 6 meses?

Primeiro Exemplo:

Por favor, tome a porção indicada como uma referência para relatar o seu consumo; por exemplo, se você tem o hábito de tomar $1 / 2$ copo de leite duas vezes ao dia, então marque que toma um copo de leite uma vez ao dia ( $1 \frac{1}{2}$ copo $+1 / 2$ copo) como no exemplo ao lado:

Segundo Exemplo:

Se você toma uma lata de refrigerante, duas a três vezes na semana, então você deve marcar como no exemplo ao lado:
Leite

Um copo ou uma xícara

( ) menos de uma vez por mês ou nunca

( ) 1-3 vezes por mês

( ) 1 vez por semana

( ) 2-4 vezes por semana

( ) 5-6 vezes por semana

(X) 1 vez por dia

( ) 2-3 vezes por dia

( ) 4 ou mais vezes por dia

Refrigerante

Uma lata ou copo

( ) menos de uma vez por mês ou nunca

( ) 1-3 vezes por mês

( ) 1 vez por semana

(X) 2-4 vezes por semana

( ) 5-6 vezes por semana

( ) 1 vez por dia

( ) 2-3 vezes por dia

( ) 4 ou mais vezes por dia

Para cada alimento listado abaixo, marque a opção que melhor descreve o seu consumo médio da quantidade especificada durante os últimos 6 meses.

1) Leite (considere leite puro ou adicionado de café, chocolate ou similares)

Um copo ou uma xícara

( ) menos de uma vez por mês ou nunca

( ) 1-3 vezes por mês

( ) 1 vez por semana

( ) 2-4 vezes por semana

( ) 5-6 vezes por semana

( ) 1 vez por dia

( ) 2-3 vezes por dia

( ) 4 ou mais vezes por dia

3) Café

Um copo ou uma xícara

( ) menos de uma vez por mês ou nunca

( ) 1-3 vezes por mês

( ) 1 vez por semana

( ) 2-4 vezes por semana

( ) 5-6 vezes por semana

( ) 1 vez por dia

( ) 2-3 vezes por dia

( ) 4 ou mais vezes por dia

5) logurte

Um copo ou pote

( ) menos de uma vez por mês ou nunca

( ) 1-3 vezes por mês

( ) 1 vez por semana

( ) 2-4 vezes por semana

( ) 5-6 vezes por semana

( ) 1 vez por dia

( ) 2 ou mais vezes por dia
2) Chá ou mate

Um copo ou uma xícara

( ) menos de uma vez por mês ou nunca

( ) 1-3 vezes por mês

( ) 1 vez por semana

( ) 2-4 vezes por semana

( ) 5-6 vezes por semana

( ) 1 vez por dia

( ) 2-3 vezes por dia

( ) 4 ou mais vezes por dia

4) Achocolatado em pó (produtos como Toddy ou Nescau)

1 colher de sopa

( ) menos de uma vez por mês ou nunca

( ) 1-3 vezes por mês

( ) 1 vez por semana

( ) 2-4 vezes por semana

( ) 5-6 vezes por semana

( ) 1 vez por dia

( ) 2 ou mais vezes por dia

6) Produtos à base de cereais (Neston, Mucilon, Farinha Láctea, sucrilhos e similares)

$1 / 2$ xícara ou 3 colheres de sopa

( ) menos de uma vez por mês ou nunca

( ) 1-3 vezes por mês

( ) 1 vez por semana

( ) 2-4 vezes por semana

( ) 5-6 vezes por semana

( ) 1 vez por dia

( ) 2 ou mais vezes por dia 